\title{
Production performance measurement and improvement based on the exchange of expert knowledge
}

\author{
H K Tönshoff, $M$ A Rotzoll \\ Institute of Production Engineering and Machine Tools (IFW) \\ Department of Production Management \\ University of Hannover \\ Schlosswender Strasse 5, 30159 Hannover, Germany \\ telephone: +49 (0)511762 2533, fax: +49(0) 5117625115 \\ email: \{toenshoff | rotzoll\}@mail.ifw.uni-hannover.de
}

\begin{abstract}
The benchmark has to be measured and compared continuously, it is a moving target. Just as important is the acquirement of knowledge to improve a nonsatisfying situation. Information from benchmark projects in which the production performance is investigated is used to draw conclusions about general dependencies which are scarcely or even unknown or just assumed. This information is used to control economical and logistical indicators of a company. Additionally, non-confidential knowledge from different experts in the area of production organisation and best practices are collected continuously, processed and transferred to people in charge of finding measures to solve optimising problems. Hereby important knowledge is gathered and spread out.
\end{abstract}

\section{Keywords}

Knowledge management, performance measurement, benchmarking, modelling tools and techniques, agile manufacturing 


\section{INTRODUCTION}

Nowadays companies are forced to become better fast in order to keep and improve their competitiveness. Nothing is more stable than change. Changing market demands and high innovation rates require adaptations in the organisation of the business processes to face lower product prices, shorter product life cycles, individualisation of customer demands, creation of new business fields etc. (North,1997). If we knew what we know - knowledge management becomes more and more important and is already seen as one of the milestones for the companies of the $21^{\text {s1 }}$ century. The adaptation and optimisation process becomes part of a control loop which always accompanies the company (Tönshoff and Rotzoll, 1997a). However, for small and medium-sized enterprises (SMEs) long-term planning is often still an event to which not enough attention is paid to. This paper describes a new approach in which the production performance of SMEs with small batch production is analysed and improved based on the exchange of expert knowledge gathered by a standardised European benchmark method. Experts from several organisations carry out a common benchmark in which they exchange their experience concerning best practises and the improvement of the companies' performance in different environments, branches and countries. The gathered knowledge is not lost after a benchmark is carried out but is transferred to further improvement projects at new companies. The exchange and use of this knowledge is explained in this paper.

\section{IMPROVEMENT OF PRODUCTION STUCTURES: STATE OF THE ART AND PROBLEMS}

The improvement of the production performance and thus the production structures is a crucial task within the production design phase. Several methods and philosophies have been developed to strengthen the production and to realise new organisational concepts. Most important among these are segmented company, fractal company, lean production, holonic manufacturing systems, new company, vital company and bionic manufacturing systems.

Performance measurement systems indicate weak areas, also in production. They are still focused on financial aspects, organisational indicators are only implemented rarely. The knowledge in a company is mainly enlarged by its employees and their experience. Knowledge from outside is not taken into account systematically. Investigations for this approach revealed that companies very often fail in assessing their own performance situation in comparison with other companies. They often only compare their actual situation to their own plans. This information gap can lead to decisions with disastrous aftereffects. Expert systems are created to collect available knowledge and to use it for planning processes. Applicable systems are limited to small units of the production, e.g. flexible manufacturing systems or NC programming systems. They do not cover larger production units. Mathematical and evolutionary search methods look for possibilities of improvement on their own. They are used in a limited number of areas where the required 
modelling effort is acceptable. Benchmarking is the structured process of learning from the practice of others (Camp, 1989, Spendolini, 1992). However, this method causes a lot of effort: Almost $75 \%$ of the time to be spent is required for the search of partners and the collection of data (Körschges, 1995).

The approach in this paper aims at collecting and compiling non-confidential knowledge (like best practices) which often is known only by few people. Companies of different branches are benchmarked by independent experts. These experts use their own knowledge and that of experts in other companies, branches and countries to improve a company's situation more successfully. A structured procedure how to model, classify, transfer and use this knowledge has been elaborated. Its application enables companies to improve their production organisation without much effort and costs.

\section{METHODOLOGY}

The described methodology focuses on improving the manufacturing and assembly organisation of small and medium sized companies with small batch production (Tönshoff et al., 1997b). The start of a company's analysis is the collection of quantitative information. A production model is used to describe all the information which cannot easily be described in a quantitative way. After the comparison of the company's data the exchange of improvement measures is ensured in the way that all companies and experts use the same transfer method (see figure 1).

In the first step company data is collected. This is carried out using a self-explaining questionnaire in which the company fills in the requested data, and a production model with which the organisation of the workshop is described. Several characteristic numbers and explanatory factors are calculated for the quantitative comparison of companies. Characteristic numbers indicate the performance of a company, explanatory factors are determined to select reference companies with equal or similar production-oriented or product-oriented or control-oriented characteristics. For each pair of characteristic number and explanatory factor it has been investigated once if there is a relation between them, using the data of a reasonably high number of companies. The interdependence of all characteristic numbers on the explanatory factors is marked and quantified in the Benchmark Analysis Sheet (BAS). This sheet then contains important information for improvement ideas. Only pairs with significant relationships can be taken into account during the following company specific benchmark. For the comparison with other companies a set of reference companies is selected from a database for each pair of characteristic number and explanatory factor if there is a relationship. Finally a quantitative profile of strengths and weaknesses of the production organisation is given. The analysis of the relations between characteristic numbers and explanatory factors is described in chapter 4.1 .

To have a transparent view of the production organisation a detailed model is made for each company using the Production Description Language (PDL) (Tönshoff and Rotzoll, 1996, Verweij et al., 1996). This tool has been developed especially for SMEs with small batch production to support the analysis and com 


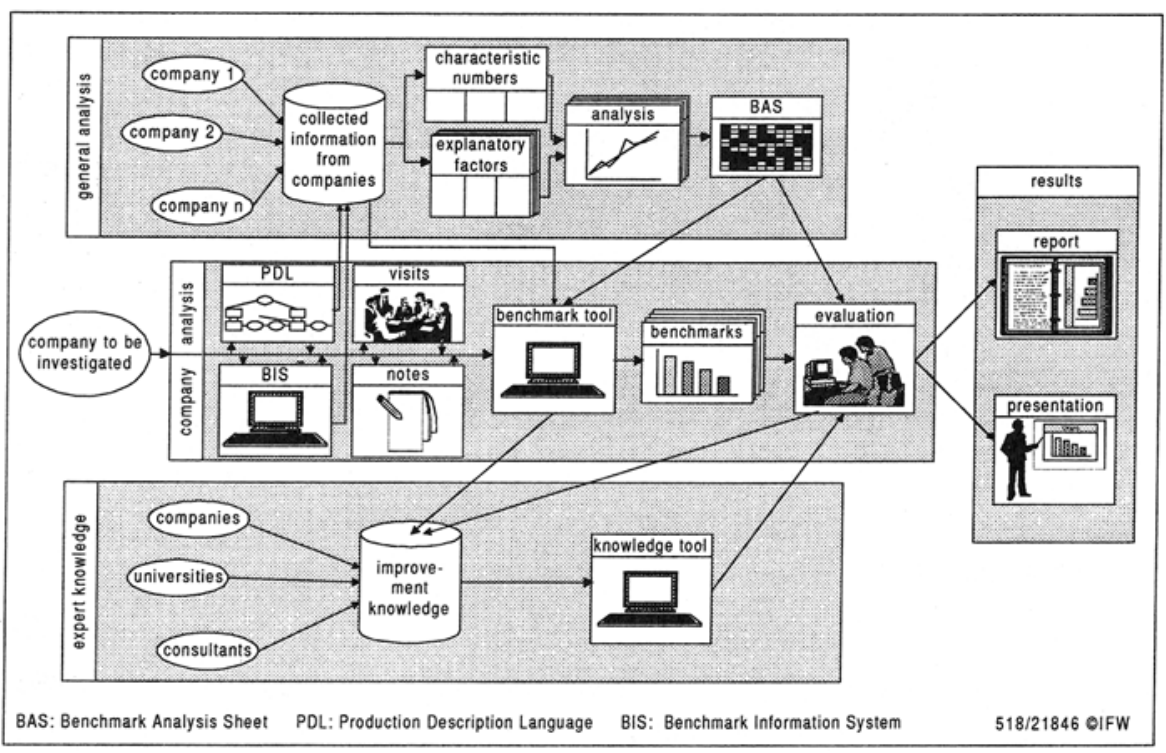

Figure 1: Methodology of knowledge-based production performance improvement

parison of production organisations. It describes the internal structure of the production and supporting units, their relationships and the material, control and information flows. A PDL picture of a production contains all relevant additional qualitative information for an improvement project which has not been collected with the questionnaire. In combination with the PDL descriptions, the production organisation is diagnosed by investigating characteristic numbers and explanatory factors.

All the benchmark information of the companies is stored in a central database. Furthermore, knowledge from experts in production organisation is collected to increase the number of possible solutions in order to improve the company's performance. The exchange of knowledge is done by sending new measures which have been applied successfully in practice to this database. The broadened knowledge then is forwarded to the improvement experts of other organisations and companies to be taken into account there. The collection and use of the expert knowledge is detailed in chapter 4.2 .

\section{KNOWLEDGE ACQUIREMENT, ANALYSIS AND USE}

Knowledge exists in many different kinds at many places in different levels of detail. In the presented approach, knowledge is collected in two different ways: on the one hand, data is gathered at the companies in a structured way. This data is analysed regarding important knowledge and relations (general analysis in figure 1). On the other hand, knowledge of experts in production organisation, such as 
experienced people from industry, university or consultants, is collected in a structured way to be used by people in charge of improving the production (expert knowledge, see figure 1).

\subsection{General analysis of interdependence}

During the analysis of a company, the characteristic numbers and explanatory factors are collected. The characteristic numbers form the basis for the benchmark. They are the indicators for the comparison of the companies. Examples for characteristic numbers are added production value per employee, the ratio of set-up times to process times, absence due to illness and percentage of customer rejects. The explanatory factors characterise the companies. They are subdivided into the three groups product-oriented, production structure-oriented and production control-oriented. Explanatory factors are e.g. number of parts per product, number of production steps, batch sizes and level of automation. Between some pairs of characteristic number and explanatory factor relations exist. E.g. the number of production steps has a significant influence on the throughput time of a product through the workshop. This information can be used to impact the performance indicators. Statistical methods have been used to find and quantify these relations.

The correlation analysis quantifies the strength of the relation of two data sets. It enables to include only those numbers into the relation model which have a significant and statistically proved impact with a reliable probability. The correlation analysis only investigates linear relations. Transferring the numbers to quadratic and root terms, also these relations can be investigated. Hereby, minima and maxima can be found. The relations obtained by the correlation analysis are mathematical relations, quantified with the correlation coefficient of Pearson. I.e. there do not necessarily have to be realistic relations but also nonsense relations could have been found. To exclude those relations that are not plausible several experts in different European countries have been asked to survey the correlation results and to eliminate unreliable relations.

To quantify the dependence of a characteristic number on the explanatory factors the univariate multiple regression analysis has been applied. For each characteristic number all explanatory factors were taken into account that had been determined with the correlation analysis and the expert investigation. Results of the regression analysis are equations for each characteristic number which describe their dependence on the explanatory factors, see equation (1).

$$
\left(\begin{array}{c}
C N_{1} \\
C N_{2} \\
\vdots \\
C N_{i}
\end{array}\right)=\left(\begin{array}{cccc}
\alpha_{1,1} & \alpha_{1,2} & \ldots & \alpha_{1, j} \\
\alpha_{2,1} & \alpha_{2,2} & \ldots & \alpha_{2, j} \\
\vdots & \vdots & \ddots & \vdots \\
\alpha_{i, 1} & \alpha_{i, 2} & \ldots & \alpha_{i, j}
\end{array}\right) \times\left(\begin{array}{c}
E F_{1} \\
E F_{2} \\
\vdots \\
E F_{j}
\end{array}\right)
$$

$\begin{array}{llll}\mathrm{CN} & \text { characteristic number } & \mathrm{i} & \text { no. of characteristic numbers } \\ \mathrm{EF} & \text { explanatory factor } & j & \text { no. of explanatory factors } \\ \alpha_{\mathrm{i}, j} & \text { coefficient of } \mathrm{EF}_{\mathrm{j}} \text { for } \mathrm{CN}_{\mathrm{i}} & & \end{array}$


Coefficients which are unequal zero have to have a probability of higher than $90 \%$ to be kept in the regression model, otherwise they are withdrawn. The variance analysis is the method to analyse this probability. Finally, when all coefficients have been analysed the degree of determination, the regression coefficient, is calculated. This coefficient describes how much of a characteristic number is influenced by the explanatory factors and how much is influenced by further factors which are not taken into account during the analysis. It varies from zero to one.

Result of these stochastic and empirical analyses is the Benchmark Analysis Sheet (BAS). It shows all characteristic numbers and explanatory factors and their interdependence. Adapting explanatory factors directly has an influence on the value of the depending characteristic numbers.

\subsection{Collection and use of expert knowledge}

To find all explanatory factors which have an impact on a characteristic number is rather difficult. Furthermore exact definitions are difficult for some factors like e.g. TQM or team work. To receive comparable information on these soft factors a detailed collection of data at a company would be necessary and cause a lot of extra effort. To avoid this, a collection of improvement measures is being carried out in Europe. Experts from different organisations like industry, university and consulting business are asked to share their knowledge and to collect measures which they already have applied in practice for improving the characteristic numbers. The start-up of the collection has been made. An internet version of the forms to fill in the measures is planned in order to simplify and speed up the knowledge collection process. The gathered knowledge is available for all people who contributed in the knowledge collection.

The knowledge is collected and stored in a structured way. Improvement measures are characterised by several items with which it is possible to fairly good assess their impact on a company's performance. The aspects collected for each measure are

- characteristic number on which the measure has a positive impact,

- strength of improvement measure,

- necessary investments and achievable savings,

- time horizon for realisation of improvement measure and

- impact on other characteristic numbers and further items.

A person in charge of optimising the production obtains a set of improvement measures from which he has to extract the appropriate ones. He can combine different measures, all aiming at improving the value of one or some characteristic numbers, and evaluate them regarding the above mentioned information using a supporting software tool. Additionally, he may use the Benchmark Analysis Sheet for further improvement ideas. Finally, he has to choose an alternative or a set of alternatives which fits best into his company. 


\section{CASE STUDY}

According to the described methodology more than 100 companies with small batch production already have been benchmarked. As an example the benchmark of a company working in the motive power engineering branch is described. The investigated company employs about 250 people and produces a lot of different metallic articles of high technical standard at a range of 150.000 units per year. The main products consist of up to four single parts being manufactured in several production steps. Customers are supplied from stock. As a result of the benchmark the company was given a comprehensive evaluation of their production structures regarding performance and efficiency. Identified strengths were e.g. low set-up times compared to process times, a high added production value per employee and a high delivery reliability. On the other side there were also some weaknesses detected. These were e.g. a low process time compared to throughput time, a low percentage of productive hours and a low stock turnover. Therefore, the company was shown various potentials for improvements in their production which have been derived from the Benchmark Analysis Sheet. The investigated company is functional-oriented, whereas the products follow similar ways through the production. Improvements are expected by changing to a more product-oriented production, which is characterised by a lower percentage of indirect employees and a shorter throughput time. Updating the production control software in use led to a more transparent planing of the production processes to avoid bottlenecks already in the long term schedule. Furthermore, measures to improve the low percentage of productive hours and to decrease the stock value are being carried out in the company.

\section{CONCLUSIONS}

The goal of the described methodology is to enable companies to improve their production performance without much effort and costs giving the people involved a broad knowledge. This knowledge is provided by the statistically proved Benchmark Analysis Sheet and several experts from different branches and countries. The knowledge base is continuously enlarged in a structured way to include new measures and trends. It contains detailed information with which the users are able to draw conclusions from for company specific improvement projects.

\section{ACKNOWLEDGEMENTS}

The investigations presented in this paper are supported within the European project 'BETTI - benchmark tool to improve the production performance', Innovation Programme, project no. IN101551. In BETTI, participants from 9 European countries investigate 100 companies within 12 months, all applying the same method described above. 
Camp, R. (1989) Benchmarking. Quality Press, Milwaukee

Körschges, A. (1995) Implementierung von Benchmarking im Unternehmen, in Benchmarking - Praxis in deutschen Unternehmen (eds. K. Mertins, G. Siebert, S. Kempf), Springer Verlag, Berlin, pp. 19-28

North, K. (1997) Wenn wir wüßten, was wir wissen: Fragen und Antworten zum Wissensmanagement, REFA Nachrichten. Vol. 50, No. 4 (August), pp. 55-60

Spendolini, M.J. (1992) The benchmarking book. American Marketing Association, New York

Tönshoff, H.K. and Rotzoll, M.A. (1996) Medium-term Analysis and Optimization of Production Structures, Annals of the German Society for Production Engineering, Vol. 3, No. 2, pp. 131-134.

Tönshoff, H.K. and Rotzoll, M.A. (1997a) Configuration of manufacturing systems with neural networks and genetic algorithms, in Proceedings of the 29th CIRP International Seminar on Manufacturing Seminars (eds. K. Iwata and K. Ueda), Osaka, 11.-13.5.1997, pp. 75-80.

Tönshoff, H.K., Rietz, W. and Rotzoll, M.A. (1997b) Improvement of production structures using benchmarking, in Managing enterprises - stakeholders, engineering, logistics and achievement (eds. D.T. Wright, M.M. Rudolph, V. Hanna, D. Gillingwater, N.D. Burns), Mechanical Engineering Publications Ltd., London, pp. 265-271.

Verweij, M.J., Rotzoll, M.A. and Müller, A. (1996) Redesign of small batch manufacturing: Basic Type Modelling, in Proceedings of the CIRP Seminars: man-. ufacturing systems, fertigungssysteme, systèmes de fabrication (ed. J. Peklenik), Faculty Press International, Ljubljana, Vol. 25, No. 3, pp. 283-287.

\section{BIOGRAPHY}

Prof. Dr.-Ing. Dr.-Ing. E.h. Hans Kurt Tönshoff, born in 1934, studied mechanical engineering at the Technical University of Hannover and took his doctor's degree in 1965. After 5 years in machine tool industry as designer and manager he became professor of the Institute of Production Engineering and Machine Tools (IFW) at the University of Hannover.

Dipl.-Ing. Martin Andreas Rotzoll, born in 1967, studied manufacturing engineering at the University of Hannover. In 1993, after his graduation, he enlarged his engineering knowledge during a research period at the Department of Mechanical Engineering, The University of Edinburgh. Since end of 1993, he is research engineer at the IFW. In 1996, he became head of the Department of Production Structures and Flows at the same institute. 